\title{
Validación del Índice Mandibular Canino en una población tumbesina
}

\author{
Approval of Mandibular Canine Index in people of Tumbes
}

Jaime Plasencia ${ }^{1}$, Kathya Segura ${ }^{2}$

http://dx.doi.org/10.21503/CienciayDesarrollo.2014.v17i1.03

\section{RESUMEN}

La odontología forense involucra el manejo, examen, evaluación y presentación de la evidencia dental en procesos civiles o criminales. La estimación del sexo en los restos óseos es muy importante para llegar a la identificación de un individuo. Los desastres conllevan a una gran pérdida de vidas humanas, que en muchos casos debido a la avanzada descomposición de los cuerpos, la falta de tiempo y recursos; tienen que ser inhumados sin la debida identificación. Sabiendo que existen métodos para identificación y determinación de edad variados pero inespecíficos para nuestra población, y siendo evidente que ningún ser humano está libre de un desastre, y que es necesario ante esta eventualidad estar preparado con datos válidos y de suficiente aplicabilidad en nuestra región; el propósito del presente estudio es validar el índice mandibular del canino (IMC) para la estimación de sexo en una población estudiantil tumbesina.

La muestra estuvo conformada por 87 hombres y 87 mujeres, estudiantes de una universidad de Tumbes, se midieron los anchos mesiodistales de caninos mandibulares y la distancia intercanina para determinar el IMC, se procesaron los datos para comparación de medias intra e intergrupos y se determinó un nuevo punto de corte.

Se concluye que las funciones discriminantes del IMC son efectivas para la estimación de sexo en la población tumbesina; así mismo el IMC ha sido validado para nuestra población, estableciendo un punto de corte único de 0,268 , con el que se logra una efectividad para estimación del sexo de hasta 90,85\%.

Palabras clave: Índice mandibular del canino, estimación de sexo.

\section{ABSTRACT}

Forensic dentistry involves the management, review, evaluation and presentation of dental evidence in civil or criminal proceedings. The estimation of the sex in the bones is very important to the identification of an individual. The disaster lead to a great loss of human lives, in many cases due to advanced decomposition of the bodies, the lack of time and resources; they have to be buried without proper identification. Knowing that there are methods for identification and determination of age varied but not specific to our population, and evident that no human being is free of a disaster, and that is necessary in this event be prepared with valid data and sufficient applicability in our region; the purpose of this study is to validate the mandibular canine index for the estimation of sex in students from Tumbes.

The sample was formed by 87 men and 87 women, students of a University of Tumbes, and it was measured the wide mesiodistal of mandibular canine and inter-canine distance to determine Mandibular Canine Index, were processed data for comparison of average intra- and intergroup and determined a new breakpoint.

It is concluded that the discriminant functions of the Mandibular Canine Index are effective for sex estimation in the population from Tumbes; likewise the Mandibular Canine Index has been validated for our population, establishing a single breakpoint of 0,268 which achieves an effectiveness for estimation of sex up to $90,85 \%$.

Key words: Mandibular Canine Index, estimation of sex.

\footnotetext{
1 Cirujano Dentista del Ministerio Público - Instituto de Medicina Legal y ciencias forenses. Doctor en Criminalística - Maestro en Estomatología.

E-mail: juxonpc@gmail.com

2 Bachiller en Estomatología UAP
} 


\section{INTRODUCCIÓN}

La odontología forense involucra el manejo, examen, evaluación y presentación de la evidencia dental en procesos civiles o criminales. La estimación del sexo en los restos óseos es muy importante para llegar a la identificación de un individuo. No siempre es fácil conseguir la determinación de los caracteres sexuales, ya que existen numerosos sujetos en los que los rasgos no están bien definidos o bien se encuentran mezcladas las características masculinas y femeninas no pudiendo afirmar con seguridad cuál es el sexo del individuo.

Una de las funciones principales de la Estomatología forense es contribuir a la identificación de personas vivas como de cadáveres, que por el avanzado estado de descomposición o producto de factores extrínsecos e intrínsecos sea imposible identificar por algún otro medio. La identificación odonto-estomatológica es de dos tipos reconstructiva, la que mediante mediciones antropométricas, exámenes auxiliares y métodos científicos pre - establecidos logra estimar edad, sexo, talla y raza; y como segundo tipo tenemos la identificación comparativa, que como su nombre lo indica nos permite comparar datos (odontograma, radiografías, fotografías intraorales) pre y post mortem a fin de establecer una identidad.

Nuestro país es propenso a diversos desastres, de causa natural u ocasionadas por el hombre (terremotos, sismos, deslizamientos de tierra, desbordes de los ríos, tsunamis, incendios, accidentes de tránsito, accidentes aéreos, etc.). Todos estos desastres conllevan a una gran pérdida de vidas humanas, que en muchos casos debido a la avanzada descomposición de los cuerpos, la falta de tiempo y recursos, tienen que ser inhumados sin la debida identificación. Otro problema actual es la situación de violencia en nuestro país con se- cuestros, asesinatos, posterior descuartizamiento y ocultamiento de restos; producto de los cuales se hace difícil la identificación de los mismos.

La región de Tumbes no es ajeno a los desastres naturales y/o antrópicos, además la delincuencia se ha ido incrementado a través de los años, lo que hace necesario tener las herramientas necesarias a fin que ante una eventualidad existan los recursos para elaborar una adecuada identificación forense (comparativa y reconstructiva).

La asignación de sexo a los restos óseos es una tarea importante e imprescindible, para ello se emplean funciones discriminantes, principalmente cuando se ha perdido la unidad y la relación anatómica del esqueleto o cuando nos enfrentamos con mandíbulas aisladas; el inconveniente que presentan las funciones discriminantes, como el índice mandibular del canino, es que se establecen para poblaciones de un área o país determinado y su extrapolación a otra realidad puede llevar a resultados no válidos, de ahí la necesidad de realizar estudios en la población tumbesina para evitar, como hasta ahora, recurrir con frecuencia a consultar series extranjeras, ya que son escasos los trabajos publicados sobre nuestra población contemporánea.

Rao NG, Rao NN, Pai ML y Kotian MS, en el año 1989, en base a un estudio, crearon el Índice Mandibular del Canino, el cual se basa en la odontometría y cuyo valor es la razón de dos medidas: el diámetro mesiodistal de la corona del canino mandibular y la otra medida es la distancia intercanina mandibular, si el valor de dicha proporción es mayor a 0,274 entonces el sexo correspondería a un sujeto masculino y si por el contrario el valor de la proporción es menor a dicha cifra $(0,274)$ entonces el sexo del individuo pertenecería al género femenino. 


\section{$\mathrm{IMC}=\underline{\text { Diámetro mesiodistal de la corona del canino inferior }}$ \\ Distancia intercanina mandibular}

\section{Punto de corte: 0,274}

Numerosos son los estudios en los que se ha pretendido validar el índice mandibular del canino o estimar su eficacia en la estimación de sexo: Padmavati (2011) determinó en una población Hindú que la eficacia para determinar sexo del IMC fue del 76\%. Vishwakarma y Guha (2011) establecieron que el canino derecho se adecuaba mejor a los propósitos del IMC. Acharya AB, Angady PV, Prabhu S y Nagnur S (2010), en la India, no encontraron utilidad a la aplicación de este índice, toda vez que la efectividad para la estimación del sexo fue del 51\%. Irfan Ahmed Mughal y Anwar Saood Saqib (2010), en Pakistán, establecieron un nuevo punto de corte de 0,250; con este nuevo punto de corte la efectividad para estimación de género fue de $71,67 \%$ para hombres y $78,72 \%$ para mujeres. Hashim y Murshid (2009) en una población Saudí, determinaron un dimorfismo de caninos en hombres y mujeres y establecieron la homogeneidad de caninos izquierdos y derechos. Ashith B y Senda Mainali (2009), en Nepal, establecieron que a pesar que las diferencias entre anchos mesiodistales de caninos y la distancia intercanina entre hombres y mujeres son significantes, al relacionar ambas medidas, dicha relación (IMC) pierde efectividad para estimar sexo, recomiendan usar el ancho mesiodistal y la distancia intercanino como funciones discriminantes de sexo. Vandana M, Susmita S y Puja B (2008), determinaron que la efectividad para estimar sexo del índice en una población de Utta Pradesh fue del $72 \%$. Ruiz Bravo (2004), en Chile, determinó el alto dimorfismo de caninos entre hombres y mujeres. Kaushal, S., Patnaik, V.V.G., Agnihotri, G. (2003), en la India, establecieron que si el ancho mesodistal del canino superaba los $7 \mathrm{~mm}$, entonces la probabilidad que el individuo fuera hombre era del $100 \%$.
Sabiendo que existen métodos para identificación y determinación de edad variados pero inespecíficos para nuestra población, y siendo evidente que ningún ser humano está libre de un desastre, y que es necesario ante esta eventualidad estar preparado con datos válidos y de suficiente aplicabilidad en nuestra región; el propósito del presente estudio es validar el Índice Mandibular del Canino para la estimación de sexo en una población estudiantil tumbesina.

\section{MATERIAL Y MÉTODOS}

Se utilizó el método empírico - analítico, el diseño de la investigación fue no experimental transversal descriptivo. La muestra estuvo conformada por 87 hombres y 87 mujeres, estudiantes de una universidad en Tumbes, cuyas edades estuvieron comprendidas entre los 17 y 25 años. Se excluyeron los alumnos que presentaban enfermedad sistémica que afecten el crecimiento y desarrollo de los dientes, alumnos con ausencia de caninos mandibulares, también aquellos alumnos con tratamiento restaurador en caninos mandibulares izquierdos.

Se utilizó un compás de dos puntas para realizar la odontometría en las unidades muestrales seleccionadas, teniendo en cuenta las normas de bioseguridad que amerita este procedimiento, se tomaron como referencia los puntos más salientes de la cara mesial y distal de caninos mandibulares, estos puntos coinciden con el diámetro máximo de la pieza (Fig. 1); para la distancia intercanina se tomaron como punto de referencia la punta de las cúspides de dichas piezas, en los casos en que se presentaron facetas de desgaste se tomó el punto medio de dicha faceta (Fig. 2). En los casos en que fue dificultoso encontrar 
adecuadamente los puntos mencionados, se procedió a tomar impresiones con alginato de la arcada mandibular, dicha impresión se vació con yeso Paris tipo IV a fin de obtener modelos de estudio donde se pueda realizar con mayor exactitud el reconocimiento de los puntos y la toma de datos correspondientes. La longitud final de las mediciones mencionadas se obtuvo al trasladar la distancia entre ambas puntas del compás a un vernier digital de alta precisión. Para la confiabilidad de los datos (fiabilidad intraexaminador), el operador pasadas 3 semanas, realizó nuevamente las mediciones en los modelos de estudio elaborados, tratando que la población re examinada sea del $20 \%$, se aplicó el coeficiente de correlación intraclase y la prueba Cohen's kappa obteniendo un coeficiente de 0,975; lo cual refleja una adecuada confiabilidad del evaluador.

Luego, con los datos obtenidos, se obtuvo el IMC para cada uno de los individuos, determinando mediante la pruebas $t$ student para muestras independientes las diferencias significativas entre los diámetros mesiodistales de los caninos izquierdos y derechos, en ambos sexos; también se establecieron las correlaciones entre sexo real y sexo obtenido mediante el IMC, para ello se utilizó la correlación de Pearson.

Finalmente, se determinó la eficiencia del Índice Mandibular del Canino en la estimación de sexo de la población estudiada y se procedió a validar dicho índice con un nuevo punto de corte (IMCt), el cual fue obtenido de la aplicación de la siguiente fórmula:

Dónde:

$\mathrm{IMCt}=[(\mathrm{IMCh}-\mathrm{sd})+(\mathrm{IMCm}+\mathrm{sd})] / 2$

IMCt $=$ Punto de corte para Índice Mandibular del Canino para población tumbesina.

$\mathrm{IMCh}=$ Media del Índice Mandibular del Canino para hombres.
IMCm= Media del Índice Mandibular del Canino en mujeres.

sd = Desviación estándar.

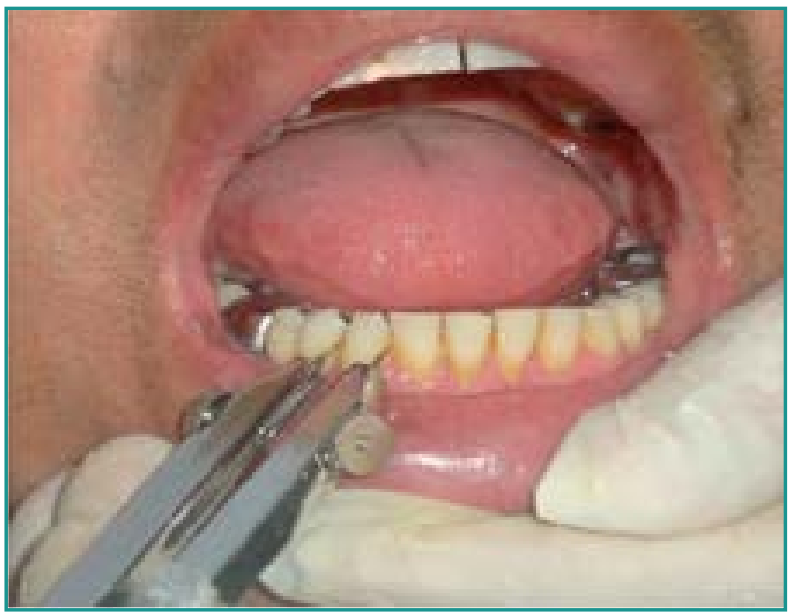

Figura 1. Medición del ancho mesiodistal de canino mandibular derecho.

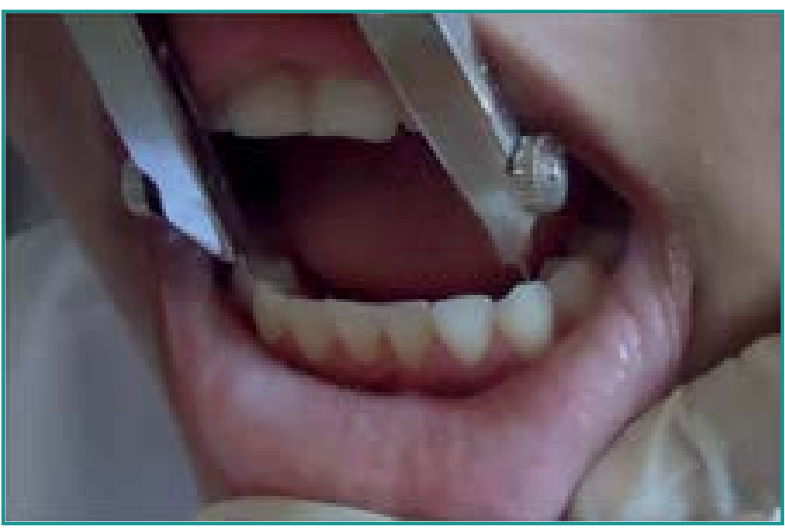

Figura 2: Medición de la distancia intercanina mandibular

\section{RESULTADOS}

Al comparar los caninos mandibulares homolaterales en hombres y mujeres, se encontraron diferencias significativas en cuanto al diámetro mesiodistal de dichos dientes tanto izquierdos como derechos, en hombres y en mujeres ( $\mathrm{p}<$ $0,05)$. Para los caninos izquierdos los valores obtenidos fueron: media del sexo masculino 7,77 $\mathrm{mm}$ y media del sexo femenino $6,45 \mathrm{~mm}$; y para los caninos derechos: media del sexo masculino $7,85 \mathrm{~mm}$ y media del sexo femenino $6,53 \mathrm{~mm}$. (Tabla 1) 
Tabla 1: Comparación de los diámetros mesiodistales de caninos mandibulares en hombres y mujeres.

\begin{tabular}{|c|c|c|c|c|c|}
\hline & Sexo real & $\mathbf{N}^{\circ}$ & Media & $\begin{array}{c}\text { Desviación } \\
\text { típ. }\end{array}$ & P valor \\
\hline DIÁMETRO & MASCULINO & 87 & 7,7682 & ,41181 & \multirow{2}{*}{$1,19 \mathrm{E}-45$} \\
\hline MD PZA 3.3 & FEMENINO & 87 & 6,4521 &, 47260 & \\
\hline DIÁMETRO & MASCULINO & 87 & 7,8494 & ,34664 & \multirow{2}{*}{$5,13 \mathrm{E}-34$} \\
\hline MD PZA 4.3 & FEMENINO & 87 & 6,5303 &, 65456 & \\
\hline
\end{tabular}

Fuente: Datos obtenidos de las unidades muestrales seleccionadas para el presente estudio.

Al comparar los anchos mesiodistales de caninos derechos e izquierdos por género, se estableció que no existen diferencias significativas en cuanto a los diámetros mesiodistales de los caninos mandibulares en hombres y mujeres ( $p>0,05)$. Para los hombres las medidas obtenidas fueron: media del diámetro del canino izquierdo 7,77 mm y media del diámetro del canino derecho $7,85 \mathrm{~mm}$. Para las mujeres los datos obtenidos fueron: media del diámetro del canino izquierdo $6,45 \mathrm{~mm}$ y media del diámetro del canino derecho $6,53 \mathrm{~mm}$. Es decir que los anchos de los caninos derechos e izquierdos en un mismo individuo son iguales. (Tabla 2)

\section{Tabla 2: Comparación de los diámetros mesiodistales de caninos mandibulares en hombres y en} mujeres por lateralidad.

\begin{tabular}{cccccc} 
DIÁMETRO & LATERALIDAD & $\mathrm{N}^{\circ}$ & Media & $\begin{array}{c}\text { Desviación } \\
\text { típ. }\end{array}$ & p valor \\
MESIODISTAL & IZQUIERDO & 87 & 7,7682 &, 41181 & \multirow{2}{*}{0,161} \\
CANINO EN & DERECHO & 87 & 7,8494 &, 34664 & \\
HOMBRES & IZQUIERDO & 87 & 6,4521 &, 47260 & \multirow{2}{*}{0,367} \\
CANINO EN & DERECHO & 87 & 6,5303 &, 65456 & \\
MUJERES & D &
\end{tabular}

Fuente: Datos obtenidos de las unidades muestrales seleccionadas para el presente estudio.

También se compararon las medias de la distancia intercanina en hombres y en mujeres obteniendo que la distancia intercanina en el género masculino es de $27,79 \mathrm{~mm}$ y la distancia inter- canina en el género femenino es de $26,12 \mathrm{~mm}$, siendo estas diferencias estadísticamente significativas $(\mathrm{p}>0,05)$. (Tabla 3$)$

Tabla 3: Comparación de la distancia intercanina en hombres y en mujeres.

\begin{tabular}{cccccc} 
& Sexo real & $\mathbf{N}^{\circ}$ & Media & $\begin{array}{c}\text { Desviación } \\
\text { típ. }\end{array}$ & P valor \\
\hline DISTANCIA & MASCULINO & 87 & 27,7877 & 1,41157 & $1,39 \mathrm{E}-10$ \\
\hline INTERCANINA & FEMENINO & 87 & 26,1210 & 1,78495 & \\
\hline
\end{tabular}

Fuente: Datos obtenidos de las unidades muestrales seleccionadas para el presente estudio. 
Luego se realizó la comparación entre el sexo real y el obtenido mediante la aplicación del IMC, para ambos caninos, los resultados indican que al utilizar el canino izquierdo, el IMC establece el sexo con una efectividad del $73,6 \%$ en hombres y del $95,4 \%$ en mujeres; y al utilizar el canino derecho la efectividad para establecer sexo en hombres fue de $86,2 \%$ y en mujeres de $93,1 \%$. (Tabla 4)

Tabla 4: Comparación de sexo real con sexo obtenido mediante la aplicación del IMC con ambos caninos.

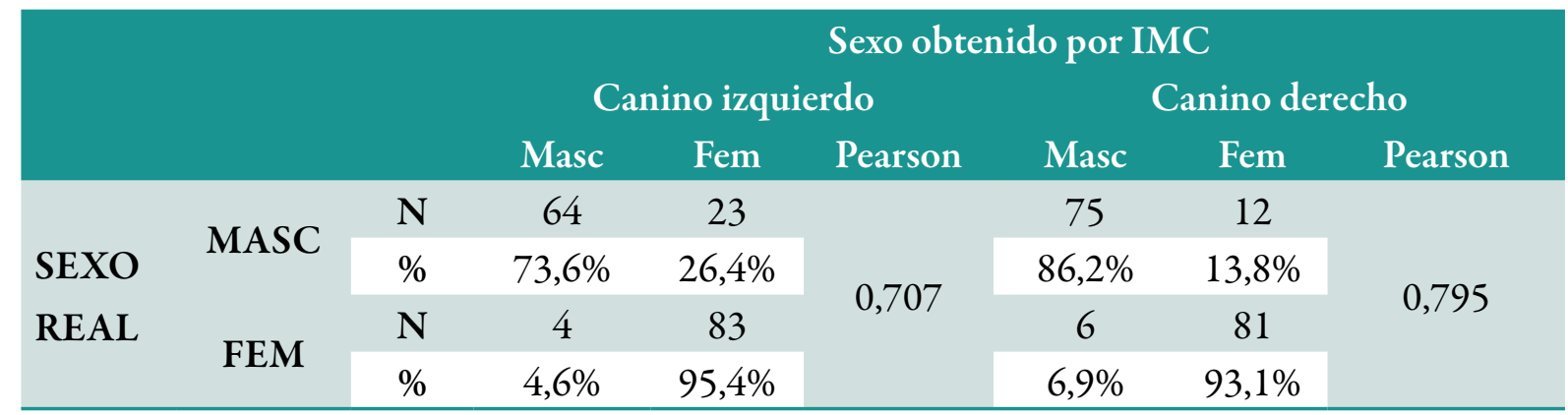

Fuente: Datos obtenidos de las unidades muestrales seleccionadas para el presente estudio.

Al realizar la prueba de Hanley y McNei para comparación de curvas ROC se estableció que no existían diferencias significativas $(\mathrm{p}>0,05)$, al estimar el sexo ya sea con el canino izquierdo o con el canino derecho. (Tabla 5)

Tabla 5: Comparación de curvas ROC del sexo obtenido al utilizar el canino derecho o el canino izquierdo.

\begin{tabular}{ccc} 
Canino utilizado & Área ROC & Valor p (Hanley y McNei) \\
\hline Izquierdo & 0,9996 & 0,3674 \\
\hline Derecho & 0,9999 & \\
\hline
\end{tabular}

Fuente: Datos obtenidos de las unidades muestrales seleccionadas para el presente estudio.

Finalmente se efectuaron las fórmulas para establecimiento del nuevo punto de corte obteniendo un nuevo punto de corte único de 0,268 , el cual tiene una efectividad para establecer el sexo de $88,55 \%$ en hombres y de $90,85 \%$ en mujeres. (Tabla 6)

Tabla 6: Establecimiento de punto de corte único para población de Tumbes.

\begin{tabular}{ccc} 
Canino utilizado & Punto de corte & Punto de corte único \\
\hline Izquierdo & 0,265 & 0,268 \\
\hline Derecho & 0,271 & 0,265 \\
\hline
\end{tabular}

Fuente: Datos obtenidos de las unidades muestrales seleccionadas para el presente estudio. 


\section{DISCUSIÓN}

El estudio establece que existen marcadas diferencias $(p<0,05)$ entre el ancho mesiodistal de caninos mandibulares entre hombres y mujeres, asimismo las diferencias de las distancias intercaninas entre ambos géneros son significativas.
También se pudo determinar que dentro de los grupos de estudio no hubieron diferencias entre el tamaño del canino, teniendo en cuenta la lateralidad del mismo, estos resultados son congruentes con lo encontrado en estudios previos. (Tabla 7)

Tabla 7: Comparación de la distancia intercanina y los diámetros mesiodistales de caninos mandibulares según diferentes estudios.

\begin{tabular}{llccccc}
\multicolumn{1}{c}{ Investigadores } & & $\begin{array}{c}\text { Kaushal } \\
(2003)\end{array}$ & $\begin{array}{c}\text { Vandana } \\
(2008)\end{array}$ & $\begin{array}{c}\text { Padmavati } \\
(2011)\end{array}$ & $\begin{array}{c}\text { Vishwakarma } \\
(2011)\end{array}$ & $\begin{array}{c}\text { Plasencia } \\
(2013)\end{array}$ \\
\hline Distancia & M & 25,873 & 26,860 & 27,220 & 25,760 & 27,788 \\
intercanina & F & 25,070 & 26,287 & 25,300 & 25,620 & 26,121 \\
Diámetro MD pza & M & 7,229 & 7,017 & -- & 7,400 & 7,849 \\
4.3 & F & 6,690 & 6,428 & -- & 6,500 & 6,530 \\
Diámetro MD pza & M & 7,299 & 7,030 & -- & 7,400 & 7,768 \\
3.3 & F & 6,693 & 6,446 & -- & 6,700 & 6,452 \\
\hline
\end{tabular}

Fuente: Antecedentes de la investigación.

El dimorfismo sexual del canino mandibular obedece a muchas teorías, la que tiene mayor aceptación y relevancia es la propuesta por Eimerl y DeVore (1965) quienes postularon que, producto de la evolución de los primates en seres humanos, una herencia genética que nos han dejado es la apariencia de agresividad que proporcionan los caninos, estos dientes al ser más grandes y al mostrarlos a sus competidores cumplían la función de intimidación y como arma de ataque y defensa, especialmente en los machos, siendo los caninos de vital importancia para la supervivencia y para la reproducción. Según su opinión, no es de extrañar entonces que, en realidad, los caninos inferiores muestran una evidente resistencia a diferentes enfermedades que afectan los tejidos calcificados y estructuras periodontales, así como un alto grado de dimorfismo sexual.

Si bien es cierto queda demostrado el dimorfismo entre las funciones discriminantes del IMC, nuestro estudio también logra aumentar en forma drástica la efectividad de dicho índice, al elaborar un nuevo punto de corte. En comparación con estudios previos se logra la mayor efectividad alcanzada hasta el momento. (Tabla 8) 
Tabla 8: Comparación de la efectividad del nuevo punto de corte encontrado.

\begin{tabular}{|c|c|c|c|c|c|c|c|}
\hline \multicolumn{2}{|c|}{ Investigadores } & $\begin{array}{c}\text { Rao } \\
(1981)\end{array}$ & $\begin{array}{l}\text { Vandana } \\
(2008)\end{array}$ & $\begin{array}{c}\text { Ahmed } \\
\text { Mughal (2010) }\end{array}$ & \multicolumn{3}{|c|}{ Plasencia (2013) } \\
\hline \multirow{2}{*}{$\begin{array}{l}\text { Punto de } \\
\text { corte }\end{array}$} & 3.3 & \multirow{2}{*}{0,274} & \multirow{2}{*}{0,256} & \multirow{2}{*}{0,250} & \multirow{2}{*}{\multicolumn{2}{|c|}{$\begin{array}{l}0,265 \\
0,271\end{array}$}} & \multirow{2}{*}{0,268} \\
\hline & 4.3 & & & & & & \\
\hline \multirow{4}{*}{ Efectividad } & \multirow{2}{*}{ M } & \multirow{2}{*}{$84,3 \%$} & & \multirow{2}{*}{$71,67 \%$} & PZA 4.3 & $89,7 \%$ & \multirow{2}{*}{$88,55 \%$} \\
\hline & & & & & PZA 3.3 & $87,4 \%$ & \\
\hline & \multirow{2}{*}{$\mathrm{F}$} & \multirow{2}{*}{$84,5 \%$} & & \multirow{2}{*}{$78,72 \%$} & PZA 4.3 & $89,7 \%$ & \multirow{2}{*}{$90,85 \%$} \\
\hline & & & & & PZA 3.3 & $92,0 \%$ & \\
\hline
\end{tabular}

Fuente: Antecedentes de la investigación

Se discrepa con lo enunciado por Kaushal (2003) en el sentido que dicho autor aseguró que si el diámetro mesiodistal del canino mandibular superaba los $7 \mathrm{~mm}$, el sexo del evaluado sería masculino; en nuestro estudio hubo muchos casos de mujeres con canino mandibular que superaba dicha magnitud. La efectividad del nuevo punto de corte $(0,268)$ encontrado en nuestro estudio fue de $88,55 \%$ para hombres y una efectividad de $90,85 \%$ para las mujeres, de esta manera se ajusta el Índice Mandibular del Canino para nuestra población, en vista que con el punto de corte 0,274 planteado por Rao se estimaba muchas más mujeres de las que en realidad había.

\section{CONCLUSIONES}

Las funciones discriminantes del Índice Mandibular Canino son efectivas para la estimación de sexo en la población tumbesina; así mismo el Índice Mandibular del Canino ha sido validado para nuestra población, estableciendo un punto de corte único de $\mathbf{0 , 2 6 8}$, con el que se logra una efectividad para estimación del sexo de hasta $90,85 \%$.

\section{RECOMENDACIONES}

Se recomienda utilizar el punto de corte único para estimación de sexo en la población tum- besina; así como utilizar el ancho mesiodistal del canino mandibular y la distancia intercanina como factores discriminantes de sexo, toda vez que las diferencias encontradas para dichas variables fueron estadísticamente significativas. Finalmente se recomienda replicar el presente estudio en otras regiones del Perú a fin de establecer el punto de corte para la población peruana.

\section{REFERENCIAS BIBLIOGRÁFICAS}

1. Acharya $A B$, Angady PV, Prabhu S, Nagnur S. Validity of the mandibular canine index (MCI) in sex prediction: Reassessment in an Indian sample. Forensic Sci Int. 2011 January 30; 204 (3): 1 - 4. Citado en PubMed; PMID: 20846802.

2. Ashith B, Mainali S. Limitations of the mandibular canine índex in sex assessment. Journal of Forensic and Legal Medicine. 2009 February; 16 (2): 67 69.

3. Caballero H. "Odontología Legal y Forense". Universidad Mayor San Marcos. Lima - Perú, 2011.

4. Eimerl S, DeVore L. The Primates, New York, Times Inc. 1965.

5. Hashim HA, Murshid ZA. Mesiodistal tooth width - A comparison between Saudi Males and Females. Egypt Dent J. 1993; 39: 343-6.

6. Moya Pueyo. Odontología Legal y Forense. Editorial Masson. Madrid 1994.

7. Mughal IA, Saqib AS, Manzur F. Mandibular 
canine index $(\mathrm{MCI})$ - its role in determining gender. Professional Med J. 2010 September; 17(3):459-63.

8. Padmavati K, Vakar M, Afroz A, Ather S. Mandibular Canine Index - A Tool for Sex Determination. Journal international of dental Antrophology. 2011 January; 1 (5): 18-20.

9. Rao G, Rao N, Pai M, Kotian M. Mandibular canine índex - a clue for establishing sex identity. Forensic Sci Int. 1989 August; 42 (3): 249-54.

10. Ruiz Bravo. Análisis del ancho mesio distal coronario y discrepancias dento-dentarias en pacientes ortodóncicos de la ciudad de Talca [tesis]. Talca - Chile. Universidad de Talca; 2004.
11. Vandana M, Susmita S, Puja B. Mandibular canine índex as a sex determinant: A study on the population of western Uttar Pradesh. 2008; 12 (2): 56-59.

12. Vishwakarma N, Guha R. A study of sexual dimorphism in permanent mandibular canines and its implications in forensic investigations. Nepal Med Coll J. 2011; 13 (2): 96 - 99. 